\title{
L'essai pamphlétaire de Fatou Diome : \\ Écrire le « je est nôtre » face aux attaques contre Marianne
}

Valérie Dusaillant-Fernandes, University of Waterloo

Que celles et ceux qui défendent une identité nationale française unique et assimilationniste se tiennent sur leur garde, Fatou Diome est là pour protéger sa vision transculturelle et fraternelle de sa patrie adoptive. Sur les plateaux de télévision et dans ses livres, elle n'a de cesse de parler d'amour et de fraternité entre les hommes et les femmes qui vivent sur les différents continents de la Terre. L'écrivaine est déterminée à défendre avec ardeur son Afrique natale qui résiste encore, dans certaines régions, aux maux économiques, politiques et sociaux tout en revendiquant haut et fort son appartenance et son dévouement profond à cette France aux visages multiples, aux politiques migratoires changeantes et à une opinion publique divisée face à l'arrivée constante d'immigrés et de réfugiés sur son sol. En effet, un sondage IFOP de 2016 effectué auprès de plus de deux mille personnes représentatives de la population française âgée de dix-huit ans et plus, montre

une position ambivalente face à l'accueil des réfugiés sur le territoire français, empreinte avant tout de craintes et d'un malaise ressenti face à l'altérité dans un contexte économique et social vécu négativement. Ce « milieu anxieux » est néanmoins très diversifié et comprend des segments de population reflétant pour certains des postures plus bienveillantes voire humanistes, pour d'autres de profondes inquiétudes, et enfin pour certains le sentiment prégnant d'être laissés pour compte. Par ailleurs, l'étude des poids de chaque groupe au sein de la population révèle que les postures les plus extrêmes, nationalistes et identitaires, sont aujourd'hui portées par une frange de la population circonscrite à moins de $20 \%$ lorsque les valeurs du multiculturalisme et la vision d'une France cosmopolite sont partagées par 30\% de la population française (présentation du sondage). (IFOP.com, résumé) $)^{1}$

Dans son dernier livre, Marianne porte plainte! (2017), Diome s'adresse sans doute à ces moins de vingt pour cent de la population à la veille des élections présidentielles de la même année, mais aussi à toutes les personnes qui pourraient oublier le passé migratoire de la France, notamment les politiciens et les polémistes médiatiques ${ }^{2}$ qui, parfois, sont issus eux-mêmes de l'immigration. L'auteure part du principe que ces personnes trahissent l'identité de Marianne qui se veut multiple, évolutive, ouverte à l'autre et fidèle à son passé historique. Marianne, un des symboles de la République laïque française, est assez souvent représentée dans la lutte et la défense 
des droits et des libertés du peuple. En fait, la liberté est représentée par les traits d'une femme dès le quatrième siècle avant Jésus Christ (Benzaken 339). À l'origine, Marianne porte le bonnet de la liberté, le pileus, le bonnet conique des esclaves affranchis romains. Au cours des dix-huitième et dix-neuvième siècles, son couvre-chef se transformera en bonnet phrygien. Dans un entretien avec Marc Abélès, Maurice Agulhon précise que la représentation anthropomorphique d'une abstraction remonte à l'Antiquité classique. En France, après la Révolution, la représentation de l'État s'est faite à travers

l'image allégorique de la République abstraite. Au-delà de cette représentation de forme ainsi conventionnelle, il y eut quelque chose de plus chaleureux qu'on pourrait appeler la personnification ; le sens rapproché de nous par l'affectivité. Il fallait non seulement représenter la République par une femme mais aussi l'aimer, avoir des sentiments pour elle et presque croire en sa réalité. Les partisans de la République l'ont perçue comme une sorte de déesse ou d'héroïne, entité à la fois humaine et surhumaine, capable d'exciter des passions positives ou négatives. (92)

Cette étude vise à examiner comment Diome utilise, dans Marianne porte plainte !, texte hybride entre l'essai et le pamphlet, certains procédés scripturaux et son expérience personnelle pour pointer du doigt les menaces idéologiques et politiques qui pèsent sur la République française, représentée par l'allégorie de Marianne, qu'elle défend face à des adversaires qui menacent ses principes fondamentaux à la veille d'une élection présidentielle. En outre, à travers cet ouvrage, elle affirme une prise de position qui est que toute nation se construit, comme le conçoit Hédi Bouraoui, dans le « trans/vasement des cultures qui se chevauchent, se croisent et s'entrecroisent, s'attirent et se repoussent » (42). Dès lors, il s'agit aussi d'observer l'expression des idées de l'écrivaine sur les questions d'identité, de transculturalité, de nationalité et d'humanité.

\section{Un peu de soi pour parler des autres}

En fait, depuis sa plus tendre enfance au début des années 1970, Diome, elle-même Sérèreniominka ${ }^{3}$, a toujours su s'imprégner des multiples identités et culturelles qui l'ont entourée comme celles de sa grand-mère sur l'île de Nodior, dans le delta du Saloum, sur la côte sud du Sénégal, puis, plus tard, celles de son mari français avec qui elle s'est installée à Strasbourg un jour de 1994. Passionnée d'écriture depuis l'âge de treize ans, c'est adulte qu'elle raconte à travers la voix des narratrices de ses romans cette première rencontre difficile avec la terre de France dans un recueil de six nouvelles intitulé La préférence nationale (2001). Entre verve sarcastique et langage coloré, Diome dénonçait déjà, à travers la fiction, ceux qui en France, notamment les 
membres du Front national, excluent ou dénigrent l'autre sous prétexte qu'elle a une autre religion ou une autre couleur de peau, ou encore un nom qui n'est pas francisé. Rejetée par la famille de son époux et désabusée par le regard que l'on porte sur elle en France, elle divorce, se consacre à ses études de doctorat et travaille pour subsister à ses besoins. La vie en France dont elle rêvait tant n'est que batailles quotidiennes pour se faire accepter par le regard de l'autre qui donne de l'importance à son existence ; enfin c'est ce qu'elle pensait. Tout est à redéfinir sur ce sol français qui la repousse, la victimise, l'agresse malgré son désir d'y appartenir et de dominer cette langue française qu'elle aime tant. Le regard qu'elle pose sur sa société d'origine et son pays d'accueil est souvent critique, mais toujours juste et bien pensé. Ainsi, dans Le ventre de l'Atlantique (2003), Diome investit une nouvelle fois la fiction pour offrir une voix à ces femmes qui restent au pays quand leur homme ou leurs fils partent en Europe imaginant un meilleur avenir. Celles qui restent en terre d'Afrique, les mères, les épouses, les filles ne sont jamais tranquilles et vivent, par des bribes de nouvelles interposées, l'aventure exilaire du proche qui, la plupart du temps, ment à sa famille pour faire bonne figure. Le constat est sans appel dans l'œuvre de Diome : cessons les rapports de domination, n'imaginons plus un Eldorado qui n'existe pas ailleurs et laissons les gens circuler librement dans le monde pour qu'ils construisent leurs rêves, leurs identités, leurs joies et leurs douleurs. Loin de se couper des autres, elle embrasse la diversité et la mobilité, cherchant dans autrui l'humanité et l'authenticité qui sont en fait « une question d'intériorité, d'état d'esprit, d'engagement, d'éthique, de moralité » (Ramond Jurney 154). Fidèle à ses valeurs et à ses idées, Diome n'oublie pas le passé, l'histoire de l'esclavage et de la colonisation, mais affirme qu'elle n’est pas « une colonisée. Senghor l'a été, pas [elle]. [Elle est] née dans un pays indépendant. [Elle] revendique donc la souveraineté pleine et entière de la liberté conquise par les pères de la négritude. Ils ont conquis cette liberté afin qu' [elle], [elle] puisse [s]e sentir libre comme n'importe quel citoyen du monde » (Diome, «Fatou Diome », n.p.). En outre, en quittant son pays natal, Diome n'a pas laissé sa culture et son identité sénégalaise derrière elle. Au contraire, elle revient deux à quatre fois par an dans son village natal et ne se sent aucunement comme une exilée puisqu'elle est partie de son plein gré (Ramond Jurney 154). Elle est cet être « additionné » par les expériences, les rencontres, les lieux, les échanges, les cultures, les couleurs, les genres sexuels et les idées : en fait, « l'identité multiple, c'est ce que je suis », répond-elle dans un entretien à Sara Buekens en 2019 (Diome et Buekens 158). C'est dans le territoire symbolique de l'écriture que l'auteure se sent un être uni, 
là où [elle] arrive à réunir [s]es deux territoires. Dans [s]es livres, il y a un morceau de France dedans comme un chocolat qui fond. Mais pour prendre son élan, il faut avoir un socle. Sans [s]a part africaine, [s]es livres n'auraient pas le goût qu'ils ont. Franco-sénégalaise, c'est plus généreux. Dans [s]es livres, [s]on Afrique et [s]on Europe ne se battent pas en duel. Elles ont déposé les armes. Elles sont obligées de dialoguer ». (Diome, « Fatou Diome », n.p.)

Ces précisions permettent de comprendre le parcours qu'a accompli Diome pour arriver à une réflexion personnelle et esthétique sur l'entre-deux ${ }^{4}$ et l'identité plurielle.

Par ailleurs, au-delà des romans, Diome utilise l'écriture comme un moyen de prendre part aux débats de son époque, prenant la plume pour exercer sa liberté d'expression, pour argumenter, montrer du doigt les « adoptés possessifs, porte-micros du white-washing » (Diome, Marianne, 23). Au cours du prologue et des six chapitres qui composent Marianne porte plainte!, elle développe plusieurs points importants de son exposé (l'identité et la nationalité, l'extrémisme, la laïcité, la mondialisation et l'éducation ${ }^{5}$ ) pour arriver à la conclusion : « pour bâtir la civilisation qui nous abrite », il faut un « nous avec les autres », pas un « nous contre les autres, chaque peuple ayant apporté ses bouts de bois, quel que soit son Dieu » (132). Alors, comment Diome s’y prendelle pour aborder de front ces questions de fond dans son essai pamphlétaire publié dans la collection « Café Voltaire » de Flammarion, collection justement à propos pour Diome puisqu'elle est « un lieu où les humeurs s'affichent, où les idées s'entrechoquent, où les goûts se révèlent » (site de l'éditeur)?

\section{L'essai pamphlétaire : une voie/voix bien choisie pour défendre Marianne}

Comme le rappelle Pascal Riendeau, l'essai est souvent « conjugué au 'je'» (32) et son originalité réside, entre autres choses, dans sa capacité à exprimer un discours original tout en « sollicitant d'autres formes et en misant sur l'hybridité » (9). Si l'essai est un « discours critique qui a son point de départ dans le réel et qui entend prouver la légitimité de son signifié par la force de son signifiant » (Belle-Ilse Létourneau 49), il est également une exploration du savoir, une façon pour un auteur de mettre en scène des idées tout en s'inscrivant dans le texte comme sujet réfléchissant» (Riendeau 10). Sans nul doute, Marianne porte plainte! relève du genre essayistique pour toutes ces raisons, mais aussi par sa « démarche intellectuelle » qui tente, comme tout essai qui se doit, de « convaincre son lecteur de la justesse de son point de vue. Son entreprise n'est pas désintéressée. Il a un message à communiquer et pour cela, il prétend influer sur l'attitude du lecteur » (Belle-Ilse Létourneau 53). En ce sens, Diome demande d'un ton déterminé et 
impératif de voter à l'unisson contre les « attaques xénophobes, racistes, sexistes, islamophobes, antisémites, homophobes » (Marianne, 44). Par des formules telles que « du calme » (15), « nous refusons un tel postulat ! » ou « halte-là ! » (82), des onomatopées (« chut ! », 29), des exhortations à s'informer, à lire avant de prendre une information pour acquise de certains politiciens (« lisez », 89 ; «lisons », 94), Diome dirige d'une manière convaincue et convaincante le lecteur pour l'amener à voter avec discernement. Cela dit, à travers l'essai de Diome on distingue le discours du combat, de l'assaut à donner avant que la bataille ne soit à jamais perdue pour toujours. Seulement, l'assaut n'est pas bien sûr physique, mais verbal ; elle cherche dans la tonitruance des mots un moyen de ridiculiser l'adversaire pour mieux l'anéantir. Sensible aux élections de 2017 qui pourraient amener Marine Le Pen au pouvoir, Diome donne à son essai une dimension plus mordante, car l'enjeu est énorme. Selon l'essayiste, le « racisme n'est pas une politique, c'est une paralysie de l'esprit » qu'il faut combattre à tous les niveaux et c'est ensemble que les citoyens français doivent lutter, «sous peine de couler ensemble dans le naufrage de La-MarineMarchande-de-Haine » (31). En ce sens, il existe dans cet essai une dimension qui relève aussi du pamphlet par certains aspects que je vais faire ressortir maintenant.

Publication de crise, le pamphlet, expliquent Michel Hastings, Cédric Passard et Juliette Rennes, se présente comme « un écrit de circonstance » où prédomine l'idée de texte incisif (5). Dès lors, l'esprit accrocheur et grinçant de l'ouvrage de Diome se traduit dès la lecture du titre qui entreprend de faire réagir d'emblée les lecteurs. Marianne porte plainte! est avant tout un cri de protestation et d'émotion partagée, marqué typographiquement par le point d'exclamation du titre, titre d'ailleurs qui est ensuite égrainé au fil des pages pas moins de seize fois comme un leitmotiv qui invite à la dénonciation et à l'action. De même, la présence abondante d'une ponctuation forte, comme le point d'exclamation, des interjections (« Ah », 22 ; «Hélas », 15, 16, 69, 77), signale l'expressivité du langage, autrement dit les marques de l'exaspération et de la déception face aux mouvements xénophobes en France. En outre, unir le prénom Marianne à l'expression «porter plainte » s'avère un coup de force, une provocation, mais aussi une bonne aubaine. En effet, cette expression, qui informe la justice qu'une infraction a été commise, pose Marianne comme la victime d'une infraction pénale telle que la diffamation, la discrimination, l'injure raciale publique. Dès lors, Diome se place alors comme son avocate. D'ailleurs, elle se complait déjà à être «l'avocate de [s]es deux mondes [français et sénégalais], à la fois partie civile et défenderesse, [elle] [s]'épuise en plaidoiries, quand [elle] plai[t] aux uns, elle] déplai[t] aux autres » (75). De 
même, dans l'entretien accordé à Buekens en 2019, ne dit-elle pas que « [s]a vie même est une plaidoirie pour l'éducation et, partout, le monde doit vaincre l'analphabétisme » (159) ? Et puis, si Buekens la considère comme «l'effigie de la défense de la tolérance multiculturelle » (157), pourquoi une effigie n'aiderait-elle pas une autre ? La violence des mots n'est pas nécessairement de la méchanceté ou de la grossièreté, constate Yves Avril (265), ce qui n'est d'ailleurs pas le cas chez Diome. On y voit plutôt « une indignation passionnée » (265) à l'encontre des discours sur l'identité nationale et la nationalité.

Le pamphlet, dit Avril, attaque «plus [ou] moins violemment, unilatéralement, un individu ou un groupe d'individus, une idée ou un système idéologique dont l'écrivain révèle, sous la pression d'une vérité urgente et libératrice, l'imposture » (265). Il s'en prend aussi au mensonge des adversaires du pamphlétaire qui sont « des individus, dotés du pouvoir institutionnel, groupés en cliques, en 'maffias'», et dont les «pseudo-valeurs sont l'exact opposé des valeurs authentiques » (Angenot 88). Diome s'en prend alors particulièrement à François Fillon et Marine Le Pen qui stigmatisent une partie de la population et refusent d'admettre, pour des raisons politiques et personnelles, que cette nation est devenue « une société plus diverse au plan ethnique, culturel et confessionnel » (Sommaire 22). Exacerbée par le refus de Fillon de considérer la France comme une société multiculturelle lors du débat de l'entre-deux-tours de la primaire de la droite en novembre $2017^{6}$, Diome prend la plume, « sa sagaie sérère » comme elle la surnomme, pour lui répondre avec le talent littéraire qu'on lui connaît. Elle dénonce le discours sur l'assimilation du candidat à la présidence et reproche aux nationalistes comme lui leur « soi-disant trop plein de repentance » et leur désir de mettre « sous le boisseau » le sujet de la colonisation (Marianne, 50). Cela dit, « le mensonge par omission ne virginise pas l'histoire ni ne corrige ses conséquences », ajoute-t-elle assénant d'un coup ses adversaires qui « prêch[ent]» cette assimilation (50).

La liberté du pamphlet, c'est d'abord celle de la liberté d'expression, de raillerie et de ton. «Française par choix, donc par amour, mais aussi par résistance » (8), l'écrivaine accuse, à la manière de la lettre ouverte d'Émile Zola dont elle suit l'exemple (30), tous les « loups qui se jettent sur l'identité nationale » (10). Elle donne de brusques coups qui visent, par une offensive logique de l'humiliation à l'œuvre en affublant les politiciens de surnoms dévalorisants avec sa plume guerrière pour protéger les « moutons » (10) vulnérables de la République qui semblent ne pas pouvoir se défendre tous seuls parce que trop assénés du même discours politique qui annihile « la possibilité d'un débat constructif» (11). À ce propos, le sobriquet, selon Ouerdia Yermeche, 
« constitue [...] un moyen d'échange ludique en ce sens qu'il doit souvent son existence à un 'jeu de créativité langagière spontané' » (97). Marc Angenot voit l'usage du sobriquet comme une des figures indirectes de l'agression visant à « déconsidérer l'adversaire » tout en cherchant « une adhésion affective, sinon même une alliance aveugle et viscérale de la part de son auditoire » (250). Ainsi, libre de jouer avec les lettres et les sens des mots, Diome transforme Marine Le Pen en « LaMarine-Marchande-de-Haine » (Marianne, 16), Nicolas Sarkozy en « Manipulateur-gesticulant» $(13,15)$, Nadine Morano en « Mégaphone de l'Est» $(20)$, Manuel Valls en « Recycleur » ou « Don Manuel Valls », François Hollande en « Tendre-Motard de l'Élysée » (38) et enfin François Fillon en «François-Fions-nous-à-Dieu » (50). Ces surnoms analogiques ${ }^{7}$ construits péjorativement reposent sur la métonymie et mettent au grand jour des comportements, des origines ou des traits de caractère excessifs de ces politiciens. Cela est normal selon Avril puisque le pamphlet montre « la petitesse et la mesquinerie » de l'adversaire, « il fait rire du ridicule de ses procédés et du grotesque de ses attitudes »(269). C'est une façon de diminuer l'ennemi pour condamner ses propos ainsi que ses faits et gestes. Toutefois, on remarque des sobriquets plus acrimonieux à l'égard des personnalités de droite et d'extrême droite, laissant ainsi transparaître le penchant politique de Diome.

« Râleuse » (Diome, Marianne, 17), elle s'emporte à ce moment-là de son pamphlet, répétant souvent «Vos papiers! Encore nos papiers! Toujours nos papiers!» ou encore «Où allez-vous ? D'où venez-vous ? Raison du séjour? C'est votre passeport? Madame, répondezmoi, que faites-vous en France » et de répondre ironiquement « La même chose que Dupont, Dupire et Durant, j'y vis » (47). On retrouve ici la forme dialogique du pamphlet où se fait entendre la voix des adversaires, ici des forces de l'ordre zélées. Diome ne mentionne jamais les corps de métiers qui contrôlent l'identité des citoyens (policiers nationaux, douaniers, gendarmes) mais préfère plutôt faire usage de la synecdoque en désignant les personnes par les lieux qu'ils occupent (« Rues, gares, aéroports », 47). Elle reproche à ces « chers pandores » de tutoyer « les colorés » avec une «moue dédaigneuse », de toiser leur victime de «leur regard effronté » et de les assommer par une « blague grasse, mazoutant les pâquerettes » $(47)^{8}$. On notera ici l'utilisation du terme « pandore », nom argotique qui désigne en général les membres de la gendarmerie nationale, et de l'expression légèrement modifiée «au ras des pâquerettes » signifiant au sens figuré «médiocre », « désolant». Il semble ici que Diome se plait à utiliser un registre familier qui 
manifeste son dégoût envers de tels comportements et infériorise ceux-là mêmes qui abusent de leur pouvoir.

Devant le délit de faciès dans la rue ou dans les aéroports, tous les Français ne sont donc pas égaux'. Diome s'interroge souvent (36) et propose des solutions avec humour. Allégeant le ton, par exemple «l'anonymat chromatique » (44) qui mettrait fin à toutes formes d'injustice et de discrimination. L'anonymat chromatique «ferait de chaque Noir, de chaque Arabe de la République, quelqu'un d'autre, pas qu'un membre du quota visible de diversité, revendiqué, exposé les jours de charité chrétienne et victime expiatoire les jours de crise ! (43). Les Français des minorités visibles peinent à trouver du travail et à être considérés comme «d'abord des citoyens »; ils ne demandent pas à être « tolérés » dans leur propre nation, mais simplement à être « respectés chez eux », clame Diome (45). Plus loin, elle se fait la porte-parole de ces « Français non caucasiens [qui] en ont assez qu'on interroge sans cesse leur identité française " (46) et apostrophe les «adoptés bien incrustés, thuriféraires de l'appartenance » en les raillant sans attendre d'eux une réplique : « Française, Français, si certains ont besoin de le lire sur la peau pour s'en convaincre, on ne va quand même pas se l'inscrire sur le front à la farine de riz ! » (47). La formule « Français non caucasiens » atteint le but visé : dénoncer la catégorisation de l'humanité par l'usage de termes racialisants. Longtemps utilisé dans un contexte médical ou dans l'imaginaire policier du dix-neuvième siècle ${ }^{10}$, le terme de « caucasien », qui définissait autrefois une catégorie raciale, n'est plus accepté en France. De même, l'emploi du terme «thuriféraire » ajoute une dimension religieuse, une connotation savamment insérée qui permet à Diome d'attaquer ces personnes. Ne serait-elle pas alors en train de dire que « chauvinisme et religion vont de pair ${ }^{11}$ ? Ce bout de phrase n'aurait d'autre objet que d'insinuer ce rapprochement.

Elle a très bien compris de la culture française que pour se faire entendre, il faut être libre de traiter de sujets qui fâchent, qui font mal, et de débattre sur tous les fronts (médias, entretiens, livres, entre autres) avec informations, statistiques et intelligence à l'appui. Lors d'un entretien en 2015, Diome s'exprimait ainsi sur le pouvoir de l'artiste face aux injustices :

Les artistes n'ont peut-être pas de pouvoir, mais ils sont fondés à rêver un autre monde possible. Ils ont surtout le devoir de dire qu'une vie vaut une vie. C'est peutêtre enfoncer des portes ouvertes, mais il y a encore des gens qui ont besoin de l'entendre. Tant qu'ils resteront sourds, nous le répéterons : une vie vaut une vie. Quelles que soient les ressources, les possessions, les carences, la pauvreté, ou la pigmentation de la peau. On ne peut pas trier les étrangers utiles et les étrangers néfastes. («Fatou Diome », n.p.) 
Contre cette racialisation du débat public sur l'identité française qui ne cesse de progresser depuis plusieurs années en France, Diome s'insurge et préfère parler des sacrifices de ces membres des colonies qui se sont battus sous le drapeau français, de la richesse de la mixité, de l'héritage culturel laissé par les vagues successives d'immigration que le tout un chacun semble avoir oublié. Elle profite de la tribune que lui donne son pamphlet, pour parler de sa famille et surtout des siens qui sont rentrés médaillés au pays sans avoir reçu de pension de la part de la France. C'est d'abord Arfang-Lang Sarr, l'oncle de sa grand-mère, parti se battre durant la Première Guerre mondiale, puis Aliou Diome, Falang Sarr, Famara Sarr, Abdou Khady Sarr, partis durant la Seconde Guerre. Diome assure que les Sérères du Sine-Saloum étaient un peuple pacifiste réputé pour sa « tradition guerrière de défense » (Marianne, 26). Cela dit, durant la colonisation, les siens « furent recrutés en masse sous le multicolore drapeau hissé par De Gaulle, qui louait leur courage » (26-27). Elle avoue que l'un des derniers anciens combattants de sa famille, Tokôr Sarr, est mort récemment « complètement oublié par la France en paix » (27), mais lui n’ayant jamais oublié le lion blanc, c'est-à-dire le général de Gaulle. Alors, par le biais de l'écriture, c'est un hommage et un appel à porter plainte contre tous ceux qui seraient capables d'oublier « ses secouristes d'hier » (29) qui sans exception ont donné leur vie à la France, quelle que soit la couleur de leur peau : « Il est vrai que la paix favorise l'oubli! Mais, contre tous les oublieux qui l'exposent au déshonneur de l'ingratitude, la belle Marianne porte plainte !» (29).

\section{Que défend Diome à travers Marianne?}

Le « je est nôtre » : identités multiples et transculturalité

Tout comme Bouraoui, Diome écrit pour unir les cultures, faisant en sorte de ne jamais en privilégier une plus qu'une autre. Giuseppina Igonetti a justement remarqué dans l'écriture de Bouraoui que «la compréhension, la tolérance et une fraternelle unicité de regard sur la multiplicité des cultures ne permettent pas au poète, quand il s'exprime, d'en laisser une l'emporte sur les autres »(14). Il me semble que Diome fait de même, dénonçant aussi autour d'elle l'exploitation du discours sur la nationalité, sur la laïcité, la passivité des uns, l'extrémisme des autres, les amalgames, et prônant le partage des richesses du monde (Marianne, 110), la force de travail des migrants (113), le besoin d'éduquer « face à toute obscurité » $(123)^{12}$. Bien placée pour comprendre à quel point le chemin de l'insertion est parsemé d'embûches, de racisme et d'efforts constants, cette Franco-Sénégalaise vivant en France depuis presque vingt-cinq ans revendique 
son droit d'entrer dans le débat sur l'identité. Dès lors, à la manière de Bouraoui, romancier et essayiste tunisien qui, dans Transpoétique, défendait le « je est nôtre » (42), c'est-à-dire un « je » qui « refuse les frontières de l'identité » (42), le « je » domien prône l'échange entre les cultures et une meilleure connaissance de l'Autre par l'éducation : « La quête du savoir ainsi commandée au croyant ne peut qu'être bénéfique au citoyen », soulève l'écrivaine (Marianne, 129). Souhaitant que l'éducation soit au centre des débats présidentiels, elle invite à penser le « vivre ensemble » en proposant que dans l'instruction civique des programmes scolaires soit inclus l'enseignement du fait religieux dans l'école laïque qui, « sans a priori », pourrait « combattre les approximations négatives ainsi que les prêches radicaux qui endoctrinent les jeunes » $(130)^{13}$. Elle suggère aussi de mieux connaître l'Autre, non pas seulement par sa cuisine (comme les divers restaurants que l'on peut voir dans les grandes villes de l'Hexagone), mais surtout par la littérature et l'histoire, rappelant en passant que «l'idéal démocratique existe en Afrique, depuis l'empereur Soundjata Keïta et la Charte de Kourougan Fouga, datée de 1222, qui comportait déjà des droits de l'homme, un code judiciaire ainsi que des règles de bonne gouvernance » (131). Elle conseille enfin de « remettre au goût du jour les grands penseurs de la culture française, ceux qui font qu'à l'étranger quand on parle de la France il y a une lueur dans le regard ( ( Fatou Diome et Cécile Guilbert», n.p.). Qu'on aime ou pas, Diome recommande des solutions peu onéreuses qui pourraient être un frein à la recrudescence de la radicalisation en France et à « la segmentation de la communauté nationale » (Marianne, 130).

Ce n'est pas à travers des personnages de roman qu'elle affirme sa prise de position, mais à travers un « je » essayistique ironique et caustique qui se fait le porte-parole de tous les laissés pour compte lors des débats, c'est-à-dire les naturalisés, aussi appelés les « différents », que les nationalistes enracinés semblent considérer comme des « citoyens de seconde zone » (43). Pour elle, l'identité nationale française est mouvante, plurielle, et dépasse les stéréotypes de la personne blanche judéo-chrétienne que propose Nadine Moreno en 2015. ${ }^{14}$ " L'identité de toute nation », affirme Diome, «ce sont d'innombrables ruisseaux, de longueurs, de saveurs et de couleurs différentes, qui versent tous dans le même fleuve » (Marianne, 18). Mais ce fleuve est loin d'être tranquille puisque la diversité apporte son lot de critiques et de détracteurs. Ceci n'est pas étonnant, selon Gilles Dupuis, puisque dans le transculturalisme, « le but ultime est de transformer l'identité de chacun à travers une longue négociation ardue et parfois douloureuse de l'altérité » $(500){ }^{15}$. Cette douloureuse négociation avec l'Autre dont parle Dupuis, la France la vit depuis près d'un 
siècle d'immigration ${ }^{16}$ au point que c'est maintenant la jeune génération, celle de ces jeunes Français issus de l'immigration de leurs arrière-grands-parents, grands-parents ou parents qui aimeraient que la reconnaissance de leur citoyenneté soit enfin non plus basée sur la couleur de leur peau ou de leurs racines, mais sur des acquis légaux. En effet, n'oublions pas que la nationalité française s'acquiert de plusieurs façons : par le droit du sang, le droit du sol et la naturalisation ${ }^{17}$. Comme le rappelle également Diome, "être français, c'est une adhésion aux valeurs de la République, qui ne regarde ni les gènes, ni le culte, ni le lieu de naissance » (Marianne, 89). Diome est de celles et ceux qui croient, comme Afef Benessaieh, qu'avec quelques exceptions, « la plupart des sociétés dans le monde sont culturellement mixtes et les frontières nationales englobent rarement des populations culturellement ou ethniquement homogènes $»(15)^{18}$. De fait, les études montrent que la France contemporaine «s'est largement construite sur l'immigration (un Français sur quatre a un grand-parent issu de l'immigration) » (Sommaire 14). Dès lors, ce n'est pas une vision multiculturelle de l'identité française qu'elle propose, mais une vision plus transculturelle. Se définissant comme niodioroise, strasbourgeoise et sénégalaise, Diome assure qu'il faut aller bien plus loin que l'acceptation et la connaissance de l'Autre : il faut qu'il y ait à la base, si l'on reprend les termes de Patrick Imbert, « la culture de la relation comme impact de l'autre sur soi et de soi sur l'autre » (28). De plus, l'image de l'identité française que suggère Diome tend à respecter les valeurs et principes de la République : la liberté, l'égalité, la fraternité, la laïcité, mais aussi la solidarité, le respect de la langue française, l'absence de toutes formes de discrimination. C'est ce point de vue que partage Marie Blaise, pour qui «la transculturalité par opposition à l'interculturalité vise bien plus qu'une acceptation et que la connaissance de l'Autre. Elle a pour objectif de transformer les représentations et les modes de penser les relations entre êtres humains en s'appuyant sur des valeurs humanistes 'égalité, liberté, fraternité' et culturelles : la laïcité et le libre arbitre » (451).

Etre libre, être humaniste : l'identité et la nationalité

C'est ensuite la liberté de la libre circulation de tout individu sur la planète que défend Diome telle qu'elle est définie «dans l'article 13 de la Déclaration universelle des droits de l'homme, adoptée le 10 décembre 1948 par les Nations Unies : '1. Toute personne a le droit de circuler librement et de choisir sa résidence à l'intérieur d'un État. 2. Toute personne a le droit de quitter son pays, y compris le sien, et de revenir dans son pays'» (Marianne, 101). Malheureusement, l'auteure se rend à l'évidence que « seuls les ressortissants des pays riches 
jouissent pleinement de cet article » (101). La libre circulation des personnes, imposée par une mondialisation économique grandissante, est inégale et elle est souvent freinée par une administration pesante, des lois contraignantes, des dictatures ou des conflits armés qui empêchent les populations de se déplacer comme bon leur semble.

En faisant référence à la Déclaration universelle des droits de l'homme, Diome semble vouloir réconcilier les perspectives humaniste et politique des droits humains, ce qui est tout à fait possible selon Pablo Gilabert. En effet, dans « Les perspectives humaniste et politique sur les droits humains ", il affirme que le discours des droits humains est fortement lié à des structures institutionnelles spécifiques. Il définit les deux perspectives de la façon suivante :

La perspective humaniste met l'accent sur le fait central qu'il y a un ensemble de droits abstraits que chacun revendique contre tout autre, et ce en vertu de leur humanité commune et non de leur appartenance à une structure institutionnelle en particulier. La perspective politique, quant à elle, permet de rendre compte du fait que les droits humains sont, et devraient être mis en œuvre dans la pratique par l'identification de droits bien plus particuliers et qui sont toujours liés à des cadres institutionnels donnés. (252)

Ces deux perspectives sont compatibles et nécessaires pour un meilleur vivre ensemble. Galibert affirme que les États-nations ou peuples déterminent

la forme des processus politiques qui ont des répercussions sur leurs intérêts fondamentaux, et qu'ils choisissent le type de mise en œuvre des droits humains le plus approprié étant donné leur culture et la mesure dans laquelle ils y sont attachés (ou du moins les aspects de cette culture qui n'entrent pas en conflit avec les droits humains). [...] L'approche humaniste s'avère particulièrement utile dans la mesure où elle nous apporte la distance critique dont nous avons besoin à l'égard des institutions politiques et des cadres culturels. Grâce à cette distance critique, nous pouvons nous apercevoir qu'il y a des interactions positives et des interactions négatives entre, d'une part, l'engagement humaniste à l'égard des droits abstraits et, d'autre part, l'exigence de respecter la diversité culturelle et les institutions incarnant une forme d'auto-détermination politique. (272)

Dès lors, Diome se fait l'écho littéraire, sans le vouloir, de la réflexion philosophique de Galibert en invoquant les principes de la Déclaration universelle des droits de l'homme. Les perspectives humaniste et politique, qui se complètent et se limitent l'une l'autre (Galibert 273), sont complexes à mettre en œuvre. Diome est consciente de cela, d'où ses nombreux rappels aux manquements aux droits de la personne.

Elle revient d'ailleurs sur le fait que durant cette campagne présidentielle de 2017, la déchéance de nationalité fut la «marotte de l'extrême droite portée au pinacle par un 
gouvernement socialiste » (Marianne, 40). Selon elle, le principe de la déchéance n'était pas égalitaire, mais raciste puisqu'elle visait seulement « les binationaux, les citoyens venus d'ailleurs, principalement des non-Blancs » (41). Pour appuyer son propos, elle fait à nouveau référence à la Déclaration universelle des droits de l'homme, puisque cette Charte concerne tous les êtres humains sans distinction et garantit, de fait le droit à la différence (politique, culturelle, religieuse, etc.) tout en préservant les droits fondamentaux. Diome rappelle que la France s'est engagée en 1948 à reconnaître dans l'article 15 de la Charte que «1. Tout individu a droit à une nationalité [et que] 2. Nul ne peut être arbitrairement privé de sa nationalité ni du droit de changer de nationalité » (41). Certes, la Déclaration n'a pas la valeur juridique d'un traité international, mais il n'en demeure pas moins que ce texte a une valeur morale à laquelle pas moins de cent-quatre-vingttreize pays membres adhèrent. Toutefois, la déchéance de nationalité en France n'est pas dans la Constitution, mais elle est régie par l'article 25 du Code civil qui prévoit qu'une personne française peut être déchue de sa nationalité, "sauf si la déchéance a pour résultat de la rendre apatride » pour un « acte qualifié de crime ou délit constituant une atteinte aux intérêts fondamentaux de la Nation » ou « un acte de terrorisme » ou alors s'est livrée au profit d'un « État étranger à des actes incompatibles avec la qualité de Français et préjudiciables aux intérêts de la France » (Légifrance, article 25) ${ }^{19}$. Sur cette question qui la touche, puisque binationale, Diome parait agacée qu'un gouvernement socialiste mené par Hollande ait pu penser à vouloir ajouter un article à la Constitution juste après les attentats de 2015. Se prononçant avec ironie sur ce discours partagé de la gauche jusqu'à l'extrême droite, Diome marque son exaspération par un « suffit » et sa colère par des impératifs qui commande au premier ministre « Don Manuel» de ne chasser « ni Maures, ni Noirs, ni métisses » (40). L'auteure invite plutôt à la réflexion : « En démocratie, même punir demande un temps de réflexion, car il s'agit de sanctionner juste et non de faire souffrir par réaction ; qui agit ainsi se rend aussi barbare que ceux dont il condamne les actes » (40).

\section{Pour conclure}

Diome démontre au fil des pages que l'identité n'est pas un concept, mais bel et bien un ensemble de plusieurs acquis. C'est une multiplicité de facteurs, de couleurs, de sentiments, complexe à appréhender et difficile à saisir qui se construit et se modifie par les contacts avec autrui. Tel un bateau qui fend le vent pour aller droit vers le port visé, l'essayiste écrit une prose mouvementée qui, dans l'épilogue du pamphlet, invite le lecteur à saisir les pagaies pour asséner 
les loups de coups. Tel Ulysse, roi d'Ithaque, vaillant guerrier de la guerre de Troie, Diome, reineécrivaine de Niodor, complète et finit son ouvrage par une exhortation à l'action. Un appel à une révolte collective est marqué par les nombreux impératifs de l'épilogue (Marianne ; « Debout », 139 ; « Mobilisons-nous », 139 ; «Réveillons-nous », 140), ainsi que le champ lexical de la guerre («pan, pan », « combattre », « combattants », « battre », 140). Elle invoque que la liberté se mérite et qu'au-delà de l'identité nationale se trouve « l'identité humaine » (124), une identité déjà défendue par les humanistes du siècle des Lumières. Dès lors, sous la véhémence de ses propos se cache non seulement une auteure fière de son identité transculturelle, mais aussi une citoyenne française déterminée à construire des passerelles entre les gens puisque le « brassage est irréversible » (116) dans le pays de Marianne où identité nationale rime avec fragilité hexagonale.

\section{Bibliographie}

Agulhon, Maurice et Marc Abélès. « Marianne dévoilée. Entretien avec Maurice Agulhon ». Terrain 15 (1990). 91-101.

Angenot, Marc. La parole pamphlétaire. Contribution à la typologie des discours modernes. Paris : Payot, « Langages et sociétés », 1982.

---. « La parole pamphlétaire ». Études littéraires 11.2 (1978). 255-264.

« Article 25 » du Code civil. Légifrance. https://www.legifrance.gouv.fr/codes/id/LEGISCTA000006150513/2020-11-28/

Avril, Yves. «Le pamphlet: essai de définition et analyse de quelques-uns de ses procédés ». Études littéraires 11.2 (1978). 265-281.

Beaucarnot, Jean-Louis. Les noms de famille et leurs secrets. Paris : Laffont, 1988.

Belle-Ilse Létourneau, Francine. «L'essai littéraire : un inconnu à plusieurs visages... ». Études littéraires 5.1 (1972). 47-57.

Benessaieh, Afef. «Multiculturalism, Interculturality, Transculturality». Amériques transculturelles - Transcultural Americas. Dir. Afef Benessaieh. Ottawa : PU d'Ottawa, « Transferts culturels/Cultural Transfers », 2010. 11-38.

Benzaken, Jean-Charles. L'allégorie de la liberté et son bonnet dans l'iconologie des monnaies et des médailles de la Révolution française (1789-1799). La Gazette des archives 146-147 (1989). 338-377. 
Blaise, Marie. « De la pluralité culturelle à la transculturalité ». Études de linguistique appliquée, 4.152 (2008). 451-462.

Bouraoui, Hédi. Transpoétique : Éloge de Nomadisme, Montréal : Mémoire d'Encrier, 2005.

Debray, Régis. « L'enseignement du fait religieux dans l’École laïque ». Rapport au ministre de l'Éducation nationale, février 2020. https://www.education.gouv.fr/l-enseignement-du-fait-religieux-dans-1-ecole-laique-12851

Diome, Fatou. « Fatou Diome 'Je suis là pour gâcher le sommeil des puissants' ». L'Humanité, 7 août 2015. https://www.humanite.fr/fatou-diome-je-suis-la-pour-gacher-le-sommeil-despuissants-581117, consulté le 1 octobre 2020.

---. Marianne porte plainte! Paris : Flammarion, 2017.

---, et Sara Buekens. « Marianne et l'identité française. Entretien avec Fatou Diome». Revue critique de fixxion française contemporaine - Critical Review of Contemporary French Fixxion 19 (2019). 156-161.

---, Mathilde Serrel et Martin Quenehen. « Fatou Diome et Cécile Guilbert : essai tricolore et satire républicaine ». France Culture, 16 mars 2017.

https://www.franceculture.fr/emissions/ping-pong/fatou-diome-cecile-guilbert-essaitricolore-et-satire-republicaine

Dupuis, Gilles. « Transculturalism and écritures migrantes ». History of Literature in Canada: English-Canadian and French-Canadian. Dir. Reingard M. Nischik. New York et Londres: Camden House, 2008. 497-508.

Flammarion. Collection « Café Voltaire ». Page résumé de la collection, https://editions.flammarion.com/Catalogue/cafe-voltaire

« François Fillon affirme son opposition à une France multiculturelle ». Le monde, 25 novembre 2016. https://www.lemonde.fr/election-presidentielle-2017/video/2016/11/25/francoisfillon-affirme-son-opposition-a-une-france-multiculturelle_5037612_4854003.html

Gilabert, Pablo. « Les perspectives humaniste et politique sur les droits humains ». Philosophiques 42.2 (2015). 251-282.

Hastings, Michel, Cédric Passard et Juliette Rennes. « Les mutations du pamphlet dans la France contemporaine ». Mots. Les langages du politique 91 (2009). 5-17.

Igonetti, Giuseppina. « L'autre dans l'œuvre poétique et romanesque de H. Bouraoui ». Hédi Bouraoui, la transpoésie. Dir. Mansour M’Henni. Tunis : Or du Temps, 1997. 11-37. 
Imbert, Patrick. « Le texte littéraire et la transculturalité ». Le transculturel et les littératures des Amériques. Le Canada et les Amériques. Ottawa: Éditions de la Chaire de recherche de l'Université d'Ottawa: Canada: enjeux sociaux et culturels dans une société du savoir, 2012. 19-56.

Lafond Desrosiers, Marianne. «Enquête sociologique sur la migration des jeunes de la communauté rurale de Dionewar, Delta du Saloum, Sénégal. Portrait de la situation et recommandations dans le cadre du projet Entreprenariat féminin et adaptation ». Centre d'initiation à la recherche et d'aide au développement durable (CIRADD), Carleton-surMer, Québec, 2013. www.ciradd.ca

« L'enseignement du fait religieux dans l'École laïque ». Ministère de l'Éducation nationale, de la jeunesse et des sports https:/www.education.gouv.fr/l-enseignement-du-fait-religieuxdans-1-ecole-laique-12851.

Leprince, Chloé. « Des 'Caucasiens' et des 'Africains' à l'école : du racisme dans les neurosciences ? ». France Culture, 10 septembre 2019.

https://www.franceculture.fr/sciences/des-caucasiens-et-des-africains-a-lecole-du$\underline{\text { racisme-dans-les-neurosciences }}$

« Les conditions et modalités de l'acquisition de la nationalité française ». Ministère de

l'Intérieur français. https://www.immigration.interieur.gouv.fr/Accueil-etaccompagnement/La-nationalite-francaise/Les-conditions-et-modalites-de-1-acquisitionde-la-nationalite-francaise

« Les Français et leurs perceptions de l'immigration, des réfugiés et de l'identité ». Sondage IFOP.com en partenariat avec More in Common, 12 juillet 2017. https://www.ifop.com/publication/les-francais-et-leurs-perceptions-de-limmigration-desrefugies-et-de-lidentite/

" 'ONPC' - Nadine Morano : La France, un pays 'de race blanche' », Le point, 27 septembre 2015. https://www.lepoint.fr/medias/onpc-nadine-morano-la-france-un-pays-de-raceblanche-27-09-2015-1968451 260.php

Ramond Jurney, Florence. « Entretien avec Fatou Diome ». Women in French Studies 18 (2010). 148-159.

Riendeau, Pascal. Méditation et vision de l'essai. Roland Barthes, Milan Kundera et Jacques Brault. Québec : Nota Bene, coll. « Littérature(s)», 2012. 
Sibony, Daniel. Entre-deux. L'origine en partage. Paris : Seuil, 1991.

Sommaire, Jean-Claude. «La crise du 'modèle français' d'intégration ». Vie sociale 4.4 (2006). $13-25$.

Toubon, Jacques. « Enquête sur l'accès aux droits. Volume 1 - Relations police/population » le cas des contrôles d'identité ». Janvier 2017. https://www.defenseurdesdroits.fr/sites/default/files/atoms/files/enquete-relations-policepopulation-final2-11012017.pdf

Wesley Johnson, G. Naissance du Sénégal contemporain. Paris : Karthala, coll. « Hommes et sociétés », 1991.

Yermeche, Ouerdia. « Le sobriquet algérien : une pratique langagière et sociale ». Insaniyat 1718 (2002). 97-110.

Notes

${ }^{1}$ Les entretiens ont eu lieu par questionnaire auto-administré en ligne du 20 au 27 septembre 2016 et deux réunions de groupe les 19 et 20 décembre 2016.

${ }^{2}$ Nicolas Sarkozy, Nadine Moreno, Emmanuel Valls, Éric Zemmour.

${ }^{3}$ Ce sont des ethnies du Sénégal habitant le delta du Saloum. Les Sérères, le plus ancien groupe ethnique du Sénégal, sont un peuple sédentaire attaché à la terre, la culture et le bétail (Wesley Johnson 24). Les Niominkas représentent un peu moins d'un pour cent de la population. En fait, « Le nom Niominkas provient de Niumi qui signifie côte, et de $N k a$, qui signifie hommes, en langue mandingue. Comme leur nom l'indique, les Niominkas sont liés à la mer » (Lafond Desrosiers 13).

${ }^{4}$ «L'entre-deux », rappelle Daniel Sibony, est « une forme de coupure-lien entre deux termes, à ceci près que l'espace de la coupure et celui du lien sont plus vastes qu'on en croit ; et que chacune de ses deux entités a toujours partie liée avec l'autre. Il n'y a pas de no man's land entre les deux, il n'y a pas un seul bord qui départage, il y a deux bords mais qui se touchent ou qui sont tels que des flux circulent entre eux » (11). Je conçois l'entre-deux domien comme ce flux perpétuel entre les cultures qui l'habitent et la définissent.

${ }^{5}$ Dans cette étude, seuls les points sur l'identité, la transculturalité et la nationalité seront abordés.

${ }^{6}$ En novembre 2016, lors d'un débat télévisé de l'entre-deux-tours de la primaire de la droite, François Fillon fait face à Alain Juppé. À la question du journaliste David Pujadas : « Est-ce que pour vous, l'avenir de la France c'est d'être une société multiculturelle ? », François Fillon répond : «La réponse est non. La France a une histoire, elle a une langue, elle a une culture. [...] Je veux que les étrangers qui viennent s'installer dans notre pays s'intègrent, s'assimilent, respectent l'héritage culturel qui est le nôtre, parce que c'est une revendication extrêmement forte qui monte du plus profond au fond de l'âme française que de conserver nos repères, nos valeurs, une forme d'identité, naturellement qui a évolué, dans un monde qui est un monde ouvert, mais qui ne doit pas disparaître » (« François Fillon », n.p.)

${ }^{7}$ Dans Les noms de famille et leurs secrets, Jean-Louis Beaucarnot catégorise les surnoms selon deux catégories : les surnoms anecdotiques et les surnoms analogiques (23).

${ }^{8}$ Le délit de faciès est confirmé en 2017 dans une enquête menée par Jacques Toubon, Défenseur des Droits, sur cinq mille personnes en France métropolitaine. L'« enquête sur l'accès aux droits. Volume 1 - Relations police/population : le cas des contrôles d'identité » révèle que, même si « pour la majorité des enquêtés, les relations police/population sont satisfaisantes, des groupes spécifiques de personnes rapportent des expériences plus contrastées » (3). Les « hommes perçus comme noirs ou arabes apparaissent cinq fois plus concernés par des contrôles fréquents. Si l'on combine ces deux critères, $80 \%$ des personnes correspondant au profil de 'jeune homme perçu comme noir ou arabe' déclarent avoir été contrôlées dans les cinq dernières années (contre 16\% pour le reste des enquêté.e.s) » (3). L'enquête confirme également des comportements en contradiction avec la déontologie des forces de sécurité. Ainsi, $80 \%$ des personnes au profil de « jeune homme perçu comme noir ou arabe » rapportent davantage de tutoiement (40\% contre $16 \%$ de 1 'ensemble), des insultes ( $21 \%$ contre $7 \%$ de l'ensemble), des brutalités (20\% contre $8 \%$ de 1 'ensemble) lors des contrôles (3). 
${ }^{9}$ Malheureusement, certains contrôles abusifs se finissent en tragédie accentuant la défiance entre la police et la population : on nommera l'affaire Zyed Benna et Bouna Traoré en 2005, morts dans un site EDF pour fuir un contrôle, le cas Théo L. grièvement blessé à la suite de violences policières en 2017 et, plus récemment, en novembre 2020, l'affaire Michel Zecler, producteur de musique noir roué de coups par quatre fonctionnaires de police entrés dans son studio après une interpellation pour défaut de port du masque (obligatoire sur la place publique en raison de la pandémie de COVID-19). Suite à cette intervention violente, les quatre policiers ont été mis en examen.

${ }^{10}$ Voir à ce propos l'article de Chloé Leprince.

${ }^{11}$ Termes que je reprends à Marc Angenot dans La parole pamphlétaire (262).

${ }^{12}$ Faute de place dans cette étude, je limite mon analyse à quelques-uns de ces points traités par Diome.

${ }^{13}$ L'idée n'est pas nouvelle en France. Ainsi, en 1989, dans un rapport destiné au ministre de l'Éducation de l'époque, Lionel Jospin, le recteur Philippe Joutard soulignait déjà l'importance d'enseigner l'histoire des religions aux élèves face à l'absence grandissante de culture religieuse. Il s'était rendu compte que cela posait problème, car de nombreux étudiants n'étaient pas préparés à l'enseignement du patrimoine artistique, littéraire et philosophique sur les bancs universitaires. Une décennie plus tard, l'écrivain et philosophe Régis Debray avait également, en 2002, envoyé un rapport au ministre de l'Éducation nationale, Jack Lang, et constatait aussi ce «maillon manquant de l'information religieuse » (3). Pour de plus amples détails, consulter le rapport intitulé «L'enseignement du fait religieux ».

${ }^{14}$ Le 26 septembre 2015, dans l'émission de télévision « On n'est pas couché » (France 2), Nadine Morano, élue au Parlement européen et ancienne ministre, a prononcé : « Nous sommes un pays judéo-chrétien - le général de Gaulle le disait -, de race blanche, qui accueille des personnes étrangères ", créant une polémique autour de ses propos. Il s'avère que cette phrase est tirée en partie d'un extrait de l'ouvrage d'Alain Peyrefitte intitulé C'était de Gaulle publié en 1994 ( ' 'ONPC' - Nadine Morano », n.p.).

15 « The ultimate aim is to transform each other's identity through a long, arduous, and sometimes painful negotiation of Otherness », je traduis.

${ }^{16}$ Jean-Claude Sommaire rappelle que la France accueille des immigrés depuis le dix-neuvième siècle pour diverses raisons : le besoin « de main-d'œuvre (dans les périodes de développement industriel et pour la reconstruction après les deux guerres mondiales)»; le besoin de "peuplement (pour combler le déficit démographique persistant»; « l'accueil de réfugiés et proscrits (Arméniens, Russes blancs, Juifs d'Europe orientale, etc.) » (14). La France a reçu aussi des flux d'immigration des «Européens de proximité (Belges, Italiens, Espagnols, Polonais, Portugais, notamment) »; des « ressortissants de l'ancien empire colonial Algériens d'abord, puis autres Maghrébins et Africains du sud du Sahara, surtout après les indépendances) dont beaucoup seront mobilisés lors des deux conflits mondiaux »; des « migrants du reste du monde (Turcs, Sri Lankais, autres Africains, etc.) » (14).

${ }^{17}$ Voir « Les conditions et modalités de l'acquisition de la nationalité française ».

18 « Most societies around the world are culturally mixed, and national boundaries rarely enclose populations that are culturally or ethnically homogeneous $»$, je traduis.

${ }^{19}$ L'article 25.1 précise que la déchéance de nationalité n'est encourue que si les faits reprochés à l'intéressé se sont produits dans le délai de dix à quinze ans (en cas de terrorisme) à compter de la date de l'acquisition de la nationalité française. 